\title{
MHD Accelerated Flow of Maxwell Fluid in a Porous Medium and Rotating Frame
}

\author{
Faisal Salah, ${ }^{1,2}$ Zainal Abdul Aziz, ${ }^{1,3}$ Mahad Ayem, ${ }^{3}$ and Dennis Ling Chuan Ching ${ }^{4}$ \\ ${ }^{1}$ UTM Centre for Industrial and Applied Mathematics, Faculty of Science, Universiti Teknologi Malaysia, \\ 81310 Johor Bahru, Johor, Malaysia \\ ${ }^{2}$ Department of Mathematics, Faculty of Science, University of Kordofan, 51111 El Obeid, Sudan \\ ${ }^{3}$ Department of Mathematical Sciences, Faculty of Science, Universiti Teknologi Malaysia, 81310 Johor Bahru, Johor, Malaysia \\ ${ }^{4}$ Department of Fundamental and Applied Sciences, Universiti Teknologi Petronas, 31750 Tronoh, Perak, Malaysia
}

Correspondence should be addressed to Zainal Abdul Aziz; abdulazizzainal@gmail.com

Received 13 June 2013; Accepted 13 August 2013

Academic Editors: K. Netocny and R. Parwani

Copyright (C) 2013 Faisal Salah et al. This is an open access article distributed under the Creative Commons Attribution License, which permits unrestricted use, distribution, and reproduction in any medium, provided the original work is properly cited.

The magnetohydrodynamic (MHD) and rotating flow of Maxwell fluid induced by an accelerated plate is investigated. The Maxwell fluid saturates the porous medium. Both constant and variable accelerated cases are considered. Exact solution in each case is derived by using Fourier sine transform. Many interesting available results in the relevant literature are obtained as the special cases of the present analysis. The graphical results are presented and discussed.

\section{Introduction}

Several fluids including butter, cosmetics and toiletries, paints, lubricants, certain oils, blood, mud, jams, jellies, shampoo, soaps, soups, and marmalades have rheological characteristics and are referred to as the non-Newtonian fluids. The rheological properties of all these fluids cannot be explained by using a single constitutive relationship between stress and shear rate which is quite different than the viscous fluids $[1,2]$. Such understanding of the non-Newtonian fluids forced researchers to propose more models of nonNewtonian fluids.

In general, the classification of the non-Newtonian fluid models is given under three categories which are called the differential, the rate, and the integral types [3]. Out of these, the differential and rate types have been studied in more detail. In the present analysis we discuss the Maxwell fluid which is the subclass of rate-type fluids which take the relaxation phenomenon into consideration. It was employed to study various problems due to its relatively simple structure. Moreover, one can reasonably hope to obtain exact solutions from Maxwell fluid. This motivates us to choose the Maxwell model in this study. The exact solutions are important as these provide standard reference for checking the accuracy of many approximate solutions which can be numerical or empirical in nature. They can also be used as tests for verifying numerical schemes that are being developed for studying more complex flow problems [4-9].

On the other hand, these equations in the non-Newtonian fluids offer exciting challenges to mathematical physicists for their exact solutions. The equations become more problematic, when a non-Newtonian fluid is discussed in the presence of $\mathrm{MHD}$ and porous medium. Despite this fact, various researchers are still making their interesting contributions in the field (e.g., see some recent studies [1-15]). Few investigations which provide the examination of non-Newtonian fluids in a rotating frame are also presented [1-19]. Such studies have special relevance in meteorology, geophysics, and astrophysics.

To the best of our knowledge, no investigation has been reported so far which discusses the accelerated flows of nonNewtonian fluids in a rotating frame. This is the objective of the present study. Here, we examine the rotating and MHD flow induced by an accelerated plate. Two explicit examples of acceleration subject to a rigid plate are taken into account. Constitutive equations of a Maxwell fluid are 
used and modified Darcy's law has been utilized. The exact solution to the resulting problem is developed by Fourier sine transform. With respect to physical applications, the graphs are plotted in order to illustrate the variations of embedded flow parameters. The mathematical results of many existing situations are shown as the special cases of the present study.

\section{Formulation of the Problem}

We choose a Cartesian coordinate system by considering an infinite plate at $z=0$. An incompressible fluid occupying the porous space is electrically conducting by exerting an applied magnetic field $B$ 。 parallel to the $z$-axis. The electric field is not taken into consideration, the magnetic Reynolds number is small, and the induced magnetic field is not accounted for. The Lorentz force $\mathbf{J} \times B_{\text {o }}$ under these conditions is equal to $-\sigma B_{0}^{2} \mathbf{V}$. Here, $\mathbf{J}$ is the current density, $\mathbf{V}$ is the velocity field, and $\sigma$ is electrical conductivity of fluid. Both plate and fluid possess solid body rotation with a uniform angular velocity $\Omega$ about the $z$-axis.

The governing equations are

$$
\begin{gathered}
\rho\left[\frac{\partial \mathbf{V}}{\partial t}+(\mathbf{V} \cdot \nabla) \mathbf{V}+2 \Omega \times \mathbf{V}+\Omega \times(\Omega \times r)\right] \\
=-\nabla p+\operatorname{div} \mathbf{S}-\sigma B_{\circ}^{2} \mathbf{V}+\mathbf{R} \\
\operatorname{div} \mathbf{V}=0
\end{gathered}
$$

in which $\rho$ is the fluid density, $r$ is a radial vector with $r^{2}=$ $x^{2}+y^{2}, p$ is the pressure, and $\mathbf{R}$ is Darcy's resistance.

The extra stress tensor $\mathbf{S}$ for a Maxwell fluid satisfies

$$
\begin{gathered}
\mathbf{T}=-p \mathbf{I}+\mathbf{S} \\
\mathbf{S}+\lambda\left[\frac{d \mathbf{S}}{d t}-\mathbf{L S}-\mathbf{S} \mathbf{L}^{T}\right]=\mu \mathbf{A}
\end{gathered}
$$

where $\mathbf{T}$ is the Cauchy stress tensor, $\mathbf{I}$ is the identity tensor, $\mathbf{L}$ is the velocity gradient, $\mathbf{A}=\mathbf{L}+\mathbf{L}^{T}$ is the first Rivlin-Eriksen tensor, $\lambda$ is the relaxation time, $\mu$ is dynamic viscosity of fluid and $d / d t$ indicates the material derivative.

According to Tan and Masuoka [4], Darcy's resistance in an Oldroyd-B fluid satisfies the following expression:

$$
\left(1+\lambda \frac{\partial}{\partial t}\right) \mathbf{R}=-\frac{\mu \phi}{k}\left(1+\lambda_{r} \frac{\partial}{\partial t}\right) \mathbf{V}
$$

where $\lambda_{r}$ is the retardation time, $\phi$ is the porosity, and $k$ is the permeability of the porous medium. For Maxwell fluid $\lambda_{r}=0$, and hence,

$$
\left(1+\lambda \frac{\partial}{\partial t}\right) \mathbf{R}=-\frac{\mu \phi}{k} \mathbf{V}
$$

We seek a velocity field of the form:

$$
\mathbf{V}=(u(z, t), v(z, t), w(z, t))
$$

which together with (2) yields $w=0$. Then, using (1) and (3) into (5) we arrive at

$$
\begin{aligned}
& \rho\left(\frac{\partial u}{\partial t}-2 \Omega v\right)=-\frac{\partial \hat{p}}{\partial x}+\frac{\partial S_{x z}}{\partial z}-\sigma B_{\circ}^{2} u+R_{x} \\
& \rho\left(\frac{\partial v}{\partial t}+2 \Omega u\right)=-\frac{\partial \hat{p}}{\partial y}+\frac{\partial S_{y z}}{\partial z}-\sigma B_{\circ}^{2} v+R_{y}
\end{aligned}
$$

where

$$
\begin{aligned}
& \left(1+\lambda \frac{\partial}{\partial t}\right) S_{x z}=\mu \frac{\partial u}{\partial z} \\
& \left(1+\lambda \frac{\partial}{\partial t}\right) S_{y z}=\mu \frac{\partial v}{\partial z}
\end{aligned}
$$

$R_{x}$ and $R_{y}$ are $x$ - and $y$-components of Darcy's resistance $R$; and $z$-component of (1) indicates that $\widehat{p} \neq \widehat{p}(z)$, and modified pressure $\widehat{p}$ is $\widehat{p}=p-(\rho / 2) \Omega^{2} r^{2}$.

Invoking (4) and (8) in (7) and then neglecting the pressure gradient, we arrive at

$$
\begin{aligned}
& \rho(1+\left.\lambda \frac{\partial}{\partial t}\right)\left(\frac{\partial u}{\partial t}-2 \Omega v\right) \\
&=\mu \frac{\partial^{2} u}{\partial z^{2}}-\sigma B_{\circ}^{2}\left(1+\lambda \frac{\partial}{\partial t}\right) u-\frac{\mu \phi}{k} u \\
& \rho\left(1+\lambda \frac{\partial}{\partial t}\right)\left(\frac{\partial v}{\partial t}+2 \Omega u\right) \\
&=\mu \frac{\partial^{2} v}{\partial z^{2}}-\sigma B_{\circ}^{2}\left(1+\lambda \frac{\partial}{\partial t}\right) v-\frac{\mu \phi}{k} v .
\end{aligned}
$$

Mathematically when the pressure gradient is ignored, the equation of motion becomes simplified and manageably solvable, and physically the fluid is still in motion as required by the boundary conditions. We have $\mathbf{T}=-p \mathbf{I}+\mathbf{S}$, and when the pressure gradient is negligible, the stress tensor $\mathbf{T}$ is equivalent to the extra stress tensor $\mathbf{S}$, portraying the fluid motion.

The initial and boundary conditions for a constant accelerated plate are

$$
\begin{gathered}
u=v=0 \quad \text { at } t=0, z>0, \\
u(0, t)=A t, \quad v(0, t)=0 \quad \text { for } t>0, \\
u, \frac{\partial u}{\partial z}, v, \frac{\partial v}{\partial z} \longrightarrow 0, \quad \text { as } z \longrightarrow \infty, t>0,
\end{gathered}
$$

where $A$ has a dimension of $L / T^{2}$. 


\section{Solution for Constant Accelerated Flow}

Setting $F=u+i v$ and introducing the following dimensionless quantities:

$$
\begin{gathered}
\xi=Z\left(\frac{A}{v^{2}}\right)^{1 / 3}, \quad \tau=t\left(\frac{A^{2}}{v}\right)^{1 / 3}, \\
G=\frac{F}{(\nu A)^{1 / 3}}, \quad \beta=\lambda\left(\frac{A^{2}}{\nu}\right)^{1 / 3}, \\
M=\frac{\sigma B_{\circ}^{2}}{\rho}\left(\frac{\nu}{A^{2}}\right)^{1 / 3}, \quad \omega=\left(\Omega \frac{\nu^{1 / 3}}{A^{2 / 3}}\right), \\
\frac{1}{B}=\frac{\phi}{k}\left(\frac{\nu^{2}}{A}\right)^{2 / 3}, \quad c=2 i \omega+M .
\end{gathered}
$$

The above problem statement reduces to

$$
\begin{gathered}
\beta \frac{\partial^{2} G(\xi, \tau)}{\partial \tau^{2}}+(1+\beta c) \frac{\partial G(\xi, \tau)}{\partial \tau}+\left(c+\frac{1}{B}\right) G(\xi, \tau) \\
=\frac{\partial^{2} G(\xi, \tau)}{\partial \xi^{2}}, \quad \xi, \tau>0, \\
G(0, \tau)=\tau, \quad \tau>0, \\
G(\xi, 0)=\frac{\partial G(\xi, \tau)}{\partial \tau}=0, \quad \xi>0, \\
G(\xi, \tau), \frac{\partial G(\xi, \tau)}{\partial \xi} \longrightarrow 0 \quad \text { as } \xi \longrightarrow \infty, \tau>0 .
\end{gathered}
$$

After using Fourier sine transform, (14) becomes

$$
\begin{gathered}
\beta \frac{\partial^{2} G_{s}(\eta, \tau)}{\partial \tau^{2}}+(1+\beta c) \frac{\partial G_{s}(\eta, \tau)}{\partial \tau}+\left(c+\frac{1}{B}+\eta^{2}\right) G_{s}(\eta, \tau) \\
=\sqrt{\frac{2}{\pi} \eta \tau, \quad \eta, \tau>0} \\
G_{s}(\eta, 0)=\frac{\partial G_{s}(\eta, \tau)}{\partial \tau}=0, \quad \eta>0
\end{gathered}
$$

in which $G_{s}(\eta, \tau)$ indicates the Fourier sine transform of $G(\xi, \tau)$.

The solution of (15) satisfying condition (16) can be expressed as

$$
\begin{aligned}
& G_{s}(\eta, \tau)= \sqrt{\frac{2}{\pi}} \frac{\eta}{\left(c+1 / B+\eta^{2}\right)} \\
& \times\left[\frac{\left(r_{3}+c\right) r_{2} e^{r_{1} \tau}-\left(r_{4}+c\right) r_{1} e^{r_{2} \tau}}{\left(c+1 / B+\eta^{2}\right)\left(r_{2}-r_{1}\right)} \beta\right. \\
&\left.+\tau-\frac{(1+\beta c)}{\left(c+1 / B+\eta^{2}\right)}\right]
\end{aligned}
$$

or

$$
\begin{aligned}
G_{s}(\eta, \tau)= & \sqrt{\frac{2}{\pi}} \frac{\eta}{\left(c+1 / B+\eta^{2}\right)} \\
\times & {\left[\frac{r_{1}^{2} e^{r_{2} \tau}-r_{2}^{2} e^{r_{1} \tau}}{\left(c+1 / B+\eta^{2}\right)\left(r_{2}-r_{1}\right)} \beta\right.} \\
& \left.+\tau-\frac{(1+\beta c)}{\left(c+1 / B+\eta^{2}\right)}\right]
\end{aligned}
$$

with

$$
\begin{aligned}
& r_{1}, r_{2}=\frac{-(1+\beta c) \pm \sqrt{(1+\beta c)^{2}-4 \beta\left(c+(1 / B)+\eta^{2}\right)}}{2 \beta} \\
& r_{3}, r_{4}=\frac{(1-\beta c) \pm \sqrt{(1+\beta c)^{2}-4 \beta\left(c+(1 / B)+\eta^{2}\right)}}{2 \beta} .
\end{aligned}
$$

Inversion of Fourier sine transform in (17) gives

$$
\begin{aligned}
& G(\xi, \tau)= \tau e^{-(\sqrt{1 / B+c}) \xi} \\
&-\frac{2}{\pi} \int_{0}^{\infty}\left[1+\beta c-\frac{r_{1}^{2} e^{r_{2} \tau}-r_{2}^{2} e^{r_{1} \tau}}{\left(r_{2}-r_{1}\right)} \beta\right] \\
& \quad \times \frac{\eta \sin (\xi \eta)}{\left(c+1 / B+\eta^{2}\right)^{2}} d \eta
\end{aligned}
$$

The above expression for hydrodynamic fluid $M=0$ in a nonporous space $1 / B=0$ is

$$
\begin{aligned}
& G(\xi, \tau)= \tau e^{-(1+i) \sqrt{\omega} \xi} \\
&-\frac{2}{\pi} \int_{0}^{\infty}\left[1+2 i \omega \beta-\frac{r_{5}^{2} e^{r_{6} \tau}-r_{6}^{2} e^{r_{5} \tau}}{\left(r_{6}-r_{5}\right)} \beta\right] \\
& \quad \times \frac{\eta \sin (\xi \eta)}{\left(2 i \omega+\eta^{2}\right)^{2}} d \eta \\
& r_{5}, r_{6}=\frac{-(1+2 i \omega \beta) \pm \sqrt{(1+2 i \omega \beta)^{2}-4 \beta\left(2 i \omega+\eta^{2}\right)}}{2 \beta} .
\end{aligned}
$$

Letting $\omega=0$ in (21) and (22), we arrive at

$$
\begin{gathered}
G(\xi, \tau)=\tau-\frac{2}{\pi} \int_{0}^{\infty}\left[1-\frac{r_{a}^{2} e^{r_{b} \tau}-r_{b}^{2} e^{r_{a} \tau}}{\left(r_{b}-r_{a}\right)} \beta\right] \frac{\sin (\xi \eta)}{\eta^{3}} d \eta \\
r_{a}, r_{b}=\frac{-1 \pm \sqrt{1-4 \beta \eta^{2}}}{2 \beta} .
\end{gathered}
$$

The velocity field $G(\xi, \tau)$ given by (21) is equivalent to that obtained by Fetecau et al. [3, Equation 25] and [5, Equation 31]. 
Result (20) for a magnetohydrodynamic viscous fluid $\lambda=$ 0 in a porous space is

$$
\begin{aligned}
G(\xi, \tau)= & \tau e^{-(\sqrt{1 / B+c}) \xi} \\
& -\frac{2}{\pi} \int_{0}^{\infty}\left[1-e^{\left(c+1 / B+\eta^{2}\right) \tau}\right] \frac{\eta \sin (\xi \eta)}{\left(c+1 / B+\eta^{2}\right)^{2}} d \eta
\end{aligned}
$$

In the above expression, if we put $c=0$ and $1 / B=0$, we obtain the equivalent solution which was obtained by Fetecau et al. [5, Equation 22],

$$
G(\xi, \tau)=\tau-\frac{2}{\pi} \int_{0}^{\infty}\left[1-e^{\eta^{2} \tau}\right] \frac{\sin (\xi \eta)}{\eta^{3}} d \eta
$$

To see the variations of embedded flow parameters in the solution expressions, Figures 1 to 5 have been displayed in order to illustrate such variations for the constant accelerated flow. Further, in each Figure, panels (a) and (b) depict the behaviours of real and imaginary parts of dimensionless velocity.

\section{Solution for Variable Accelerated Flow}

In this section, the problem statement consists of (9) to (10), (12) and

$$
u(0, t)=\Re t^{2}, \quad v(0, t)=0 \quad \text { for } t>0,
$$

where $\mathfrak{R}$ has a dimension of $L / T^{3}$.

The flow problem after defining in terms of the dimensionless quantities,

$$
\begin{array}{cc}
\zeta=z\left(\frac{\mathfrak{R}}{\nu^{3}}\right)^{1 / 5}, & \delta=t\left(\frac{\mathfrak{R}^{2}}{\nu}\right)^{1 / 5}, \\
S=\frac{F}{\left(\nu^{2} \mathfrak{R}\right)^{1 / 5}}, \quad P=\lambda\left(\frac{\mathfrak{R}^{2}}{\nu}\right)^{1 / 5}, \\
H=\frac{\sigma B_{\circ}^{2}}{\rho}\left(\frac{v}{\mathfrak{R}^{2}}\right)^{1 / 5}, \quad \psi=\left(\Omega \frac{\nu^{1 / 5}}{\mathfrak{R}^{2 / 5}}\right), \\
\frac{1}{L}=\frac{\phi}{k}\left(\frac{\nu^{3}}{\mathfrak{R}}\right)^{2 / 5}, \quad D=2 i \psi+H,
\end{array}
$$

becomes

$$
\begin{gathered}
P \frac{\partial^{2} S(\zeta, \delta)}{\partial \delta^{2}}+(1+P D) \frac{\partial S(\zeta, \delta)}{\partial \delta}+\left[D+\frac{1}{L}\right] S(\zeta, \delta) \\
=\frac{\partial^{2} S(\zeta, \delta)}{\partial \zeta^{2}} ; \quad \zeta, \delta>0, \\
S(0, \delta)=\delta^{2}, \quad \delta>0, \\
S(\zeta, \delta), \frac{\partial S(\zeta, \delta)}{\partial \zeta} \longrightarrow 0 \quad \text { as } \zeta \longrightarrow \infty ; \delta>0, \\
S(\zeta, 0)=\frac{\partial S(\zeta, \delta)}{\partial \delta}=0, \quad \zeta>0 .
\end{gathered}
$$

Following similar methodology of solution as in the previous section, we obtain

$$
\begin{gathered}
S(\zeta, \delta) \\
=\left[\begin{array}{l}
\delta^{2}-\frac{\zeta[\delta(1+P D)+P]}{\sqrt{1 / L+D}} \\
\left.+\frac{\zeta(1+P D)^{2}(1+\zeta \sqrt{1 / L+D})}{4(\sqrt{1 / L+D})^{3}}\right] e^{-(\sqrt{1 / L+D}) \zeta} \\
+\frac{4}{\pi} \int_{0}^{\infty}\left[\left(r_{7} r_{10} e^{r_{8} \delta}-r_{8} r_{9} e^{r_{7} \delta}\right.\right. \\
\left.+\left(r_{7} e^{r_{8} \delta}-r_{8} e^{r_{7} \delta}\right)\left(D+P D^{2}-\frac{1}{L}-\eta^{2}\right)\right) \\
\left.\times\left(r_{8}-r_{7}\right)^{-1} P\right] \\
+\frac{\eta D}{\pi} \int_{0}^{\infty}\left[\frac{\left(e^{r_{8} \delta}-e^{r_{7} \delta}\right)}{r_{8}-r_{7}} P\right] \frac{\eta \sin (\zeta \eta)}{\left(D+1 / L+\eta^{2}\right)^{2}} d \eta
\end{array}\right.
\end{gathered}
$$

where

$$
\begin{aligned}
& r_{7}, r_{8}=\frac{-(1+P D) \pm \sqrt{(1+P D)^{2}-4 P\left(D+1 / L+\eta^{2}\right)}}{2 P}, \\
& r_{9}, r_{10}=\frac{(1-P D) \pm \sqrt{(1+P D)^{2}-4 P\left(D+1 / L+\eta^{2}\right)}}{2 P} .
\end{aligned}
$$

The previous expression for hydrodynamic fluid in a nonrotating $D=0$ and nonporous space $1 / L=0$ is

$S(\zeta, \delta)$

$$
\begin{aligned}
&= \delta^{2}-\frac{4}{\pi}(\delta-P)+\frac{4}{\pi} \int_{0}^{\infty} \frac{\eta \sin (\zeta \eta)}{\eta^{3}} d \eta \\
&+\frac{4}{\pi} \int_{0}^{\infty} \frac{\eta \sin (\zeta \eta)}{\eta^{5}} d \eta \\
&+\frac{4}{\pi} \int_{0}^{\infty}\left[\frac{r_{11} r_{14} e^{r_{12} \delta}-r_{12} r_{13} e^{r_{11} \delta}+\left(r_{11} e^{r_{12} \delta}-r_{12} e^{r_{11} \delta}\right)}{r_{12}-r_{11}} P\right] \\
& \times \frac{\eta \sin (\zeta \eta)}{\eta^{3}} d \eta
\end{aligned}
$$




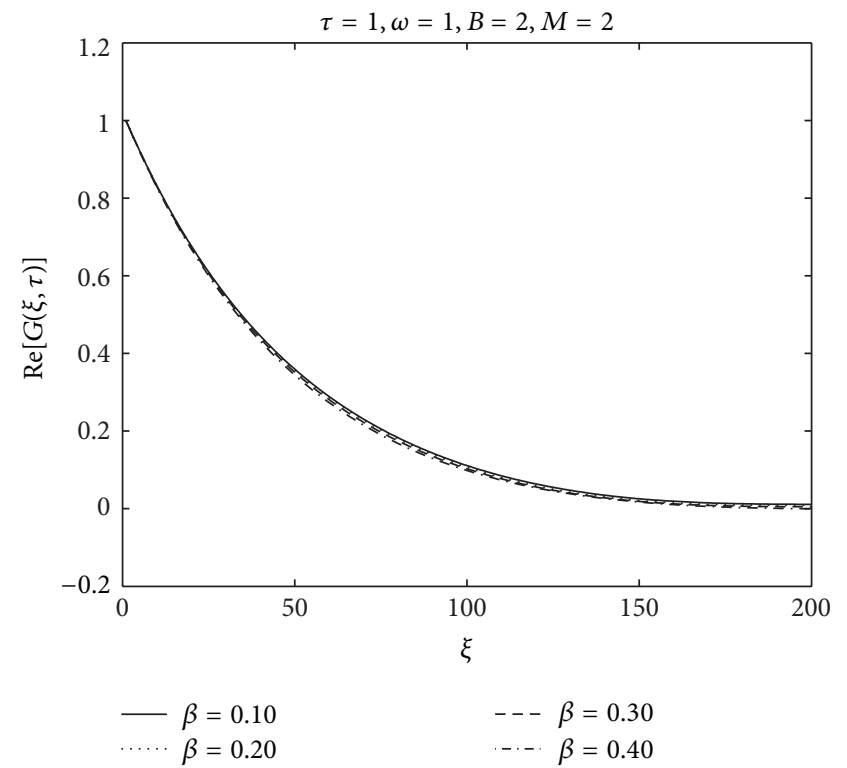

(a)

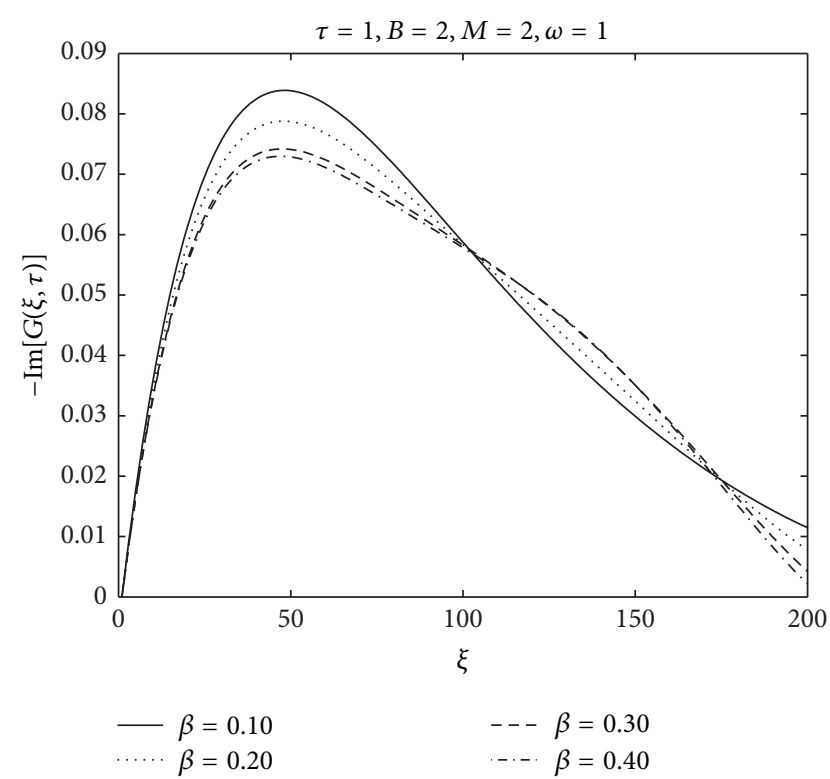

(b)

FIGURE 1: Velocity profiles for different values of $\beta$.

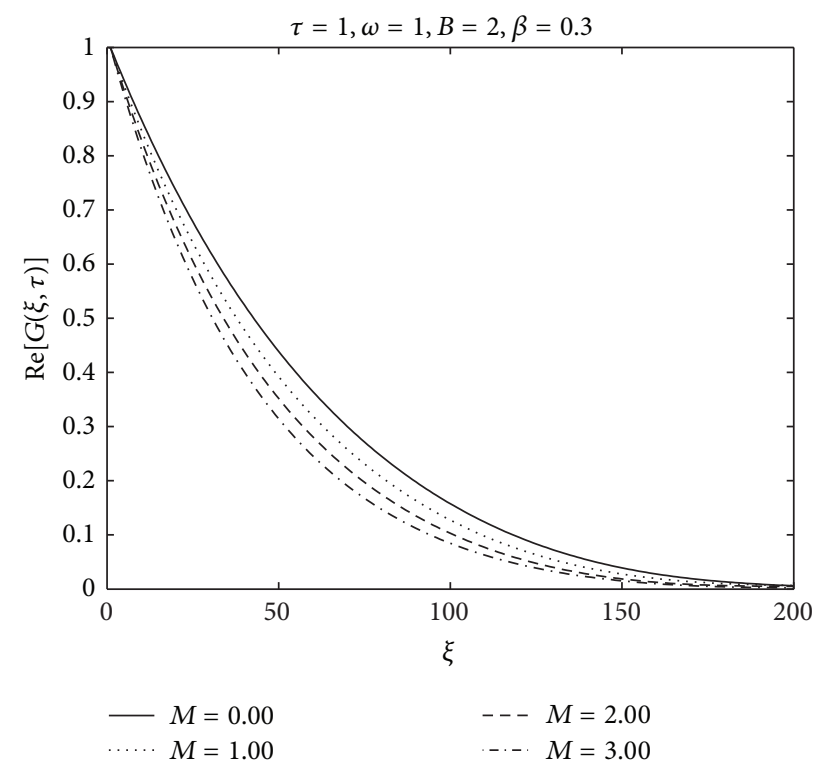

(a)

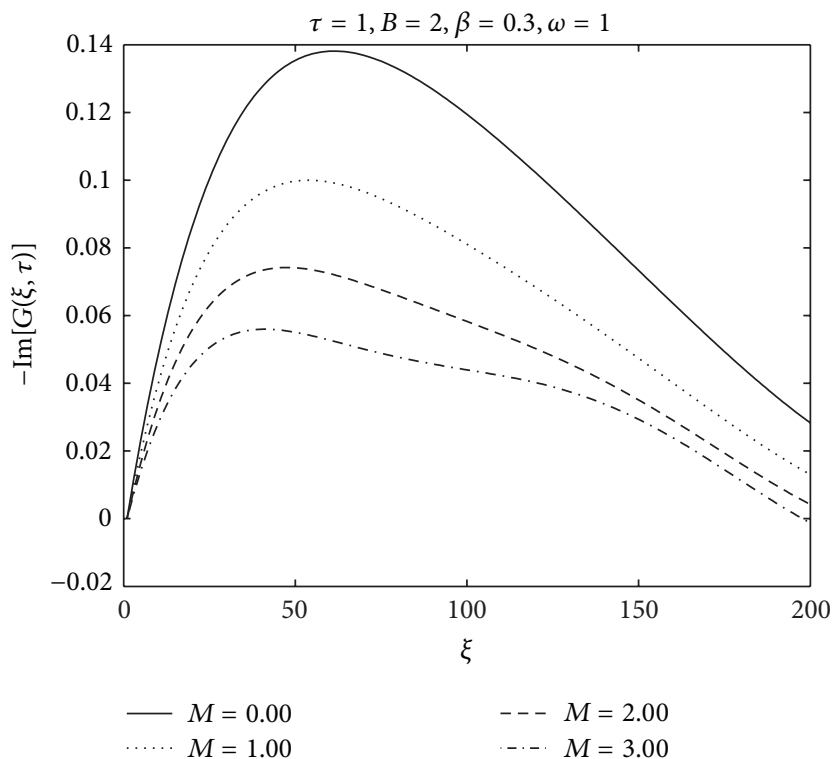

(b)

Figure 2: Velocity profiles for different values of $M$.

where

$$
\begin{gathered}
r_{11}, r_{12}=\frac{-1 \pm \sqrt{1-4 P \eta^{2}}}{2 P}, \\
r_{13}, r_{14}=\frac{1 \pm \sqrt{1-4 P \eta^{2}}}{2 P} .
\end{gathered}
$$

For a magnetohydrodynamic viscous fluid $\lambda=0 \rightarrow P=$ 0 in a porous space, (31) takes the form

$$
\begin{aligned}
S(\zeta, \delta)= & \delta^{2} e^{-(\sqrt{1 / L+D}) \zeta} \\
& +\frac{4}{\pi} \int_{0}^{\infty}\left[1-e^{\left(D+1 / L+\eta^{2}\right)}\right] \frac{\eta \sin (\xi \eta)}{\left(D+1 / L+\eta^{2}\right)^{3}} d \eta \\
& -\frac{4 \delta}{\pi} \int_{0}^{\infty} \frac{\eta \sin (\xi \eta)}{\left(D+1 / L+\eta^{2}\right)^{2}} d \eta .
\end{aligned}
$$




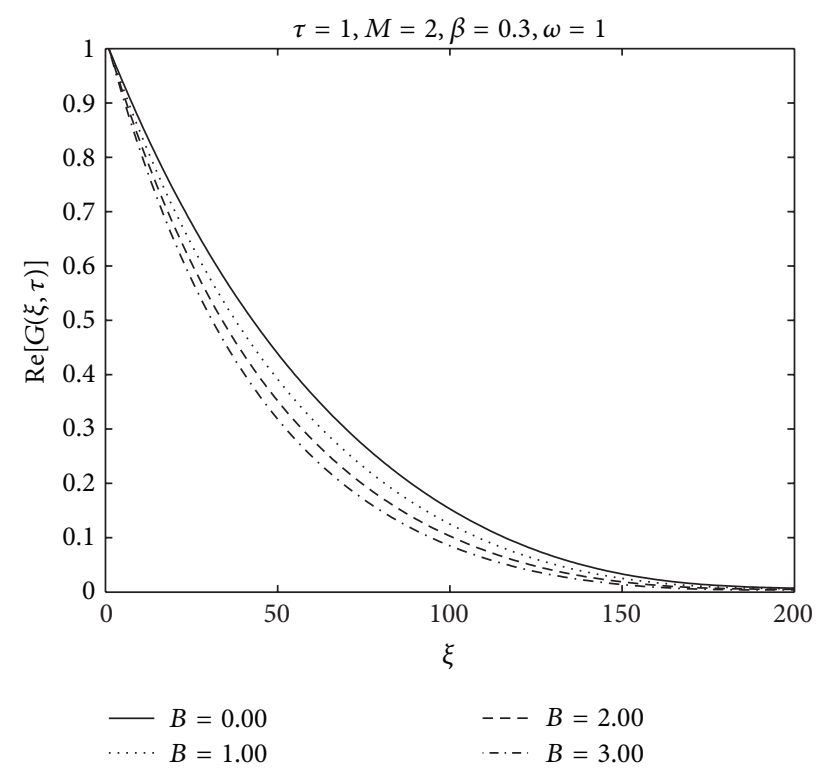

(a)

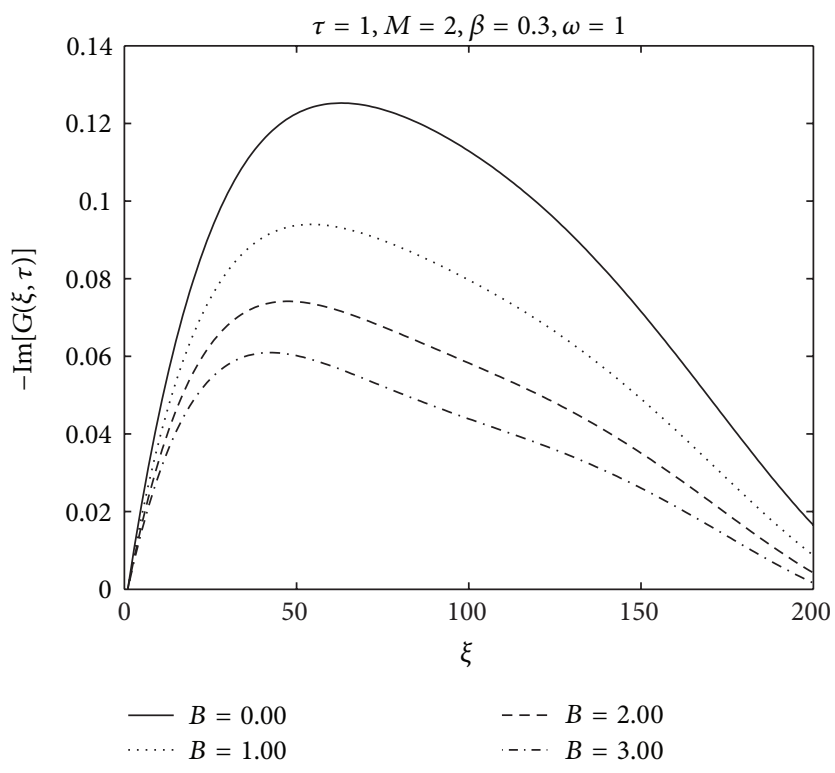

(b)

Figure 3: Velocity profiles for different values of $B$.

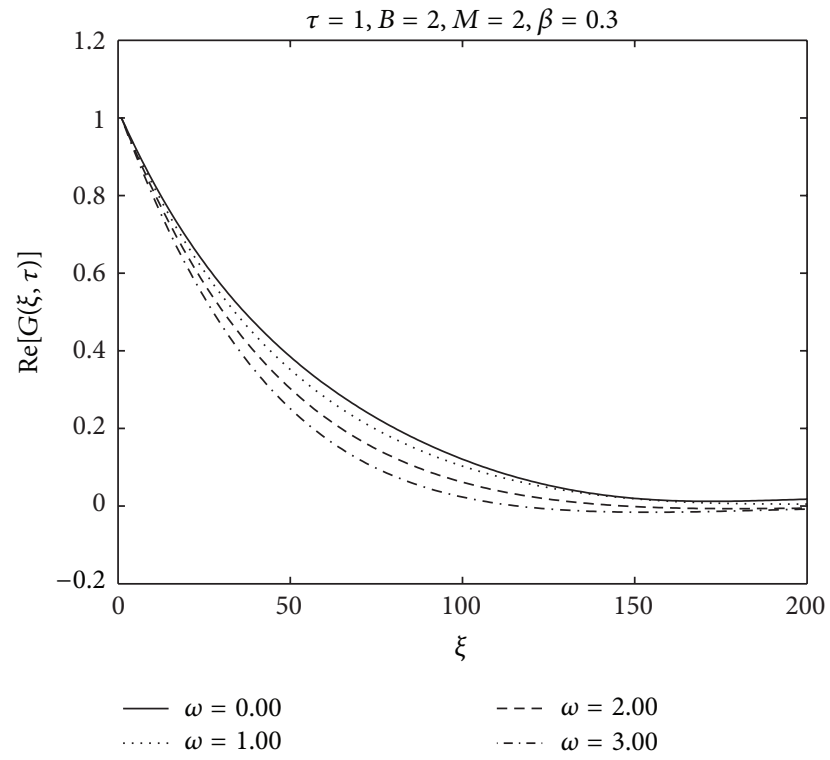

(a)

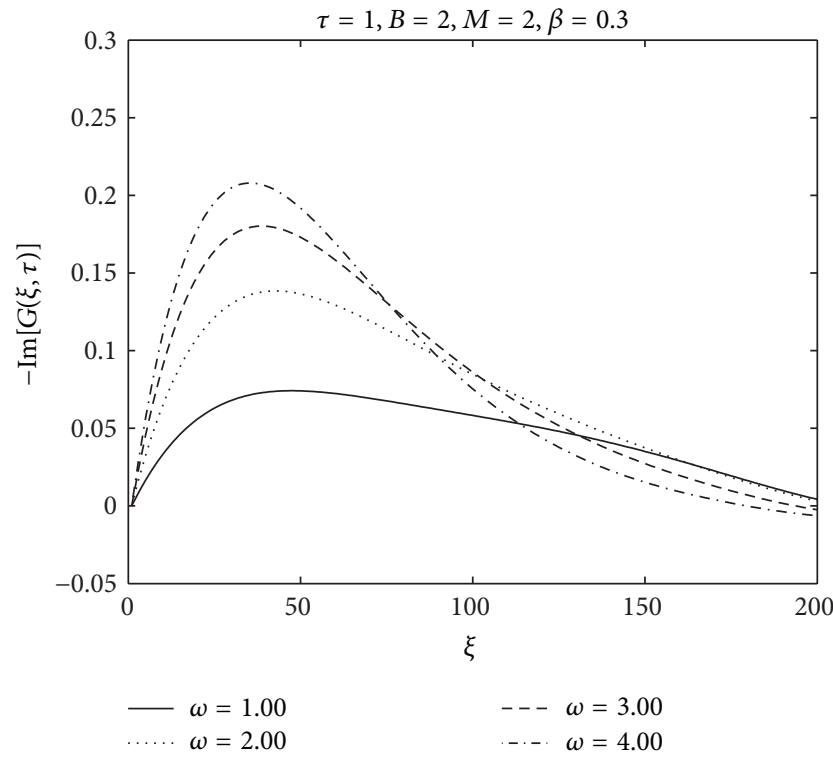

(b)

FIGURE 4: Velocity profiles for different values of $\omega$.

In order to see the variations of embedded flow parameters in the solution expressions, Figures 6, 7, 8, 9, and 10 have been displayed in order to illustrate such variations for the variable accelerated flow. Further, in each Figure, panels (a) and (b) depict the behaviours of real and imaginary parts of dimensionless velocity.

\section{Results and Discussion}

This section concerns the variations of embedded flow parameters in the solution expressions. Hence, Figures 1-10 have been displayed in order to illustrate such variations. We note that Figures 1-5 have been sketched for the constant accelerated flow, whereas Figures 6-10 are shown for the variable accelerated flow. Further, in each Figure, panels (a) and (b) depict the behaviours of real and imaginary parts of dimensionless velocity.

The effect of dimensionless relaxation time parameter $\beta$ on the velocity profile is shown in Figure 1. It is noticed too that the magnitudes of an imaginary component of velocity are decreasing function of $\beta$, and thus $r_{1}, r_{2}, r_{3}$, and $r_{4}$ in $G(\xi, \tau)$. Figure 2 elucidates the behaviour of $M$ on the 


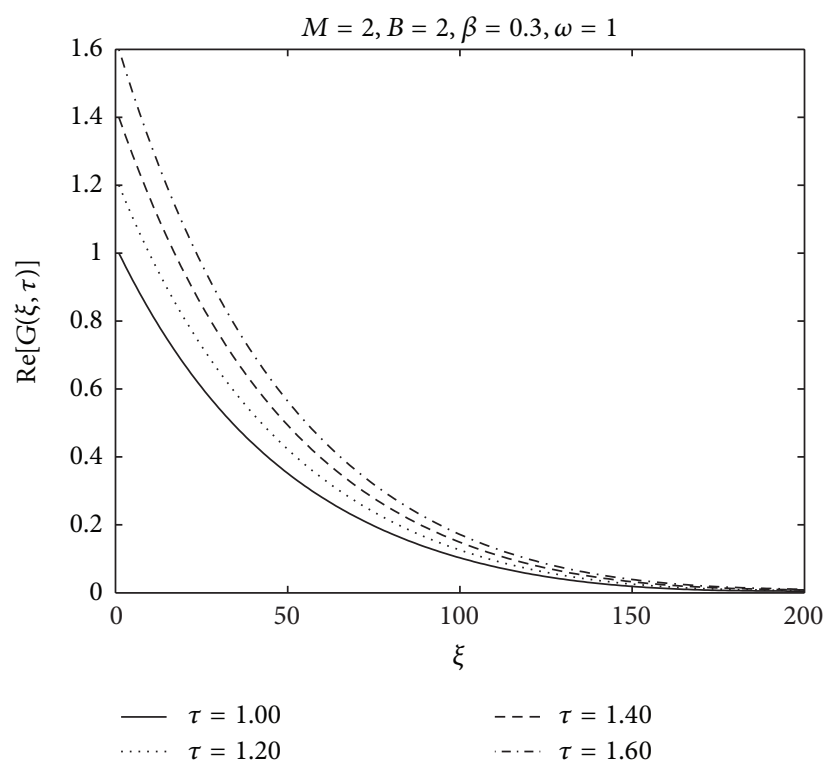

(a)

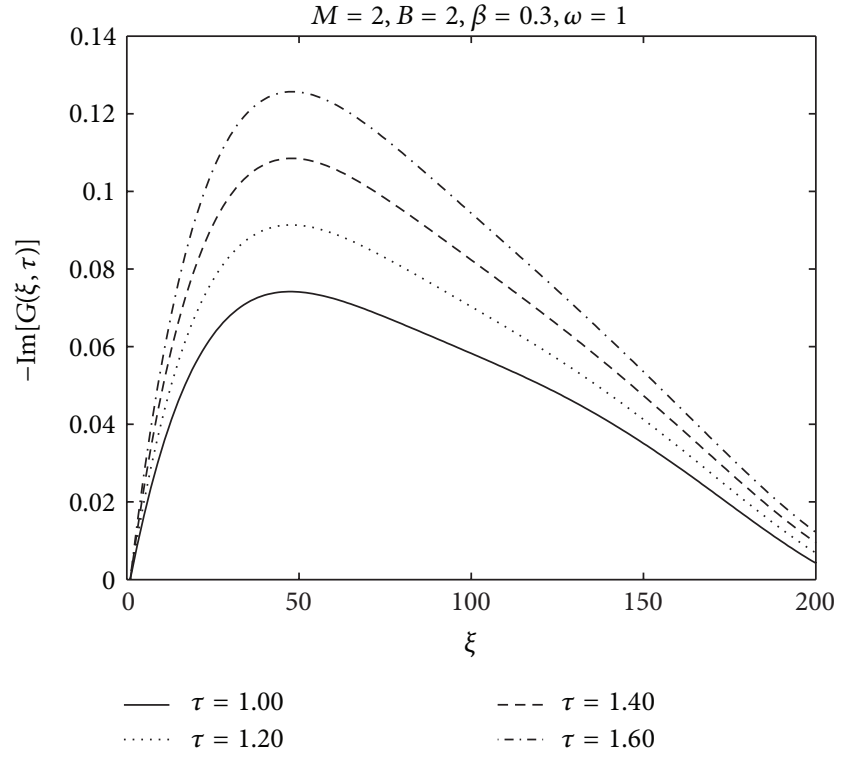

(b)

FIgURE 5: Velocity profiles for different values of $\tau$.

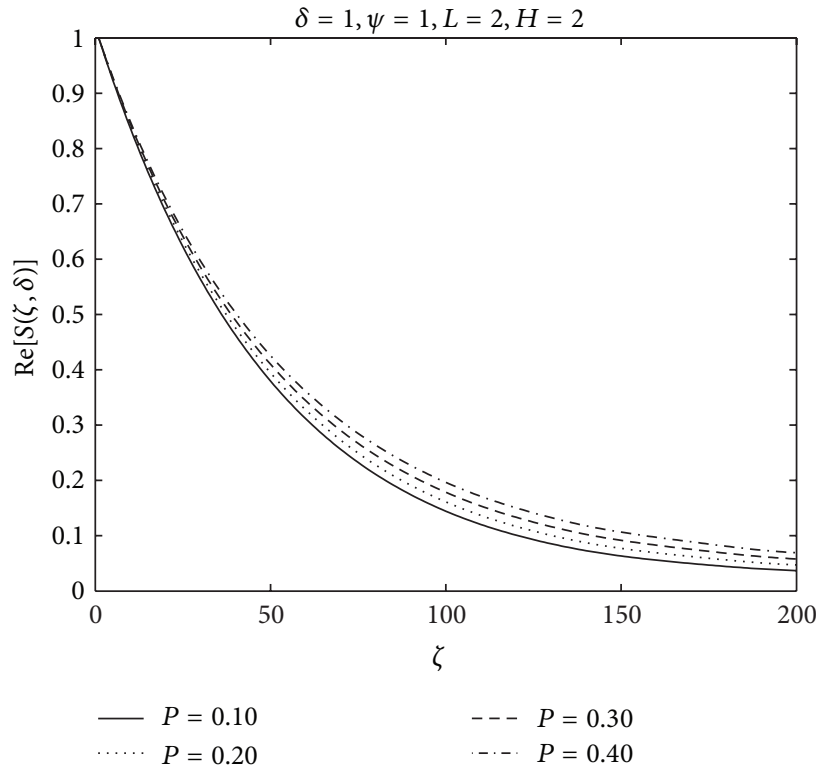

(a)

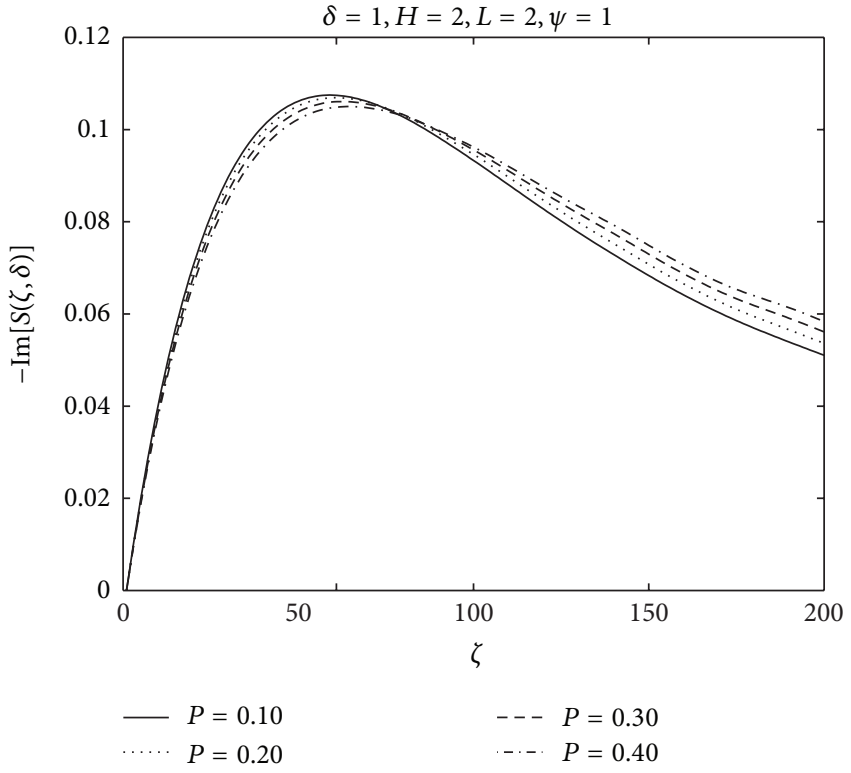

(b)

Figure 6: Velocity profiles for different values of $P$.

velocity components. It is observed that the role of $M$ on the magnitude of velocity component is qualitatively similar to that of $\beta$. This is in accordance with the fact that the Lorentz force acts as a resistance force to the flow under consideration. On the other hand, the boundary layer thickness decreases when both $\beta$ and $M$ increase. The effects of $B$ and the velocity components are sketched in Figure 3. Clearly the magnitude of velocity decreases when $B$ is increased; that is, when the medium becomes less porous (porosity $\phi$ decreases). Figure 4 plots the effects of the solid body rotation in terms of $\omega$. Figure 4(a) indicates that the real part of velocity is reduced when $\omega$ increases. However, Figure 4(b) shows correspondingly a different behaviour, whereby the velocity first increases and then decreases with respect to $\omega$. It is seen that the variation here is more oscillatory in character. The variation of $\tau$ on the magnitude of velocity components is 


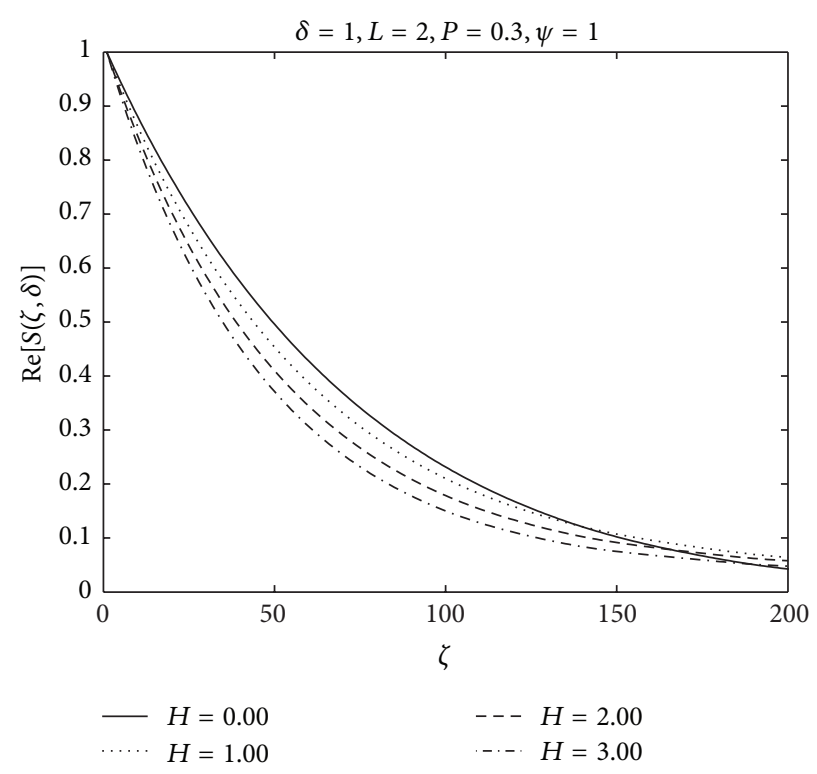

(a)

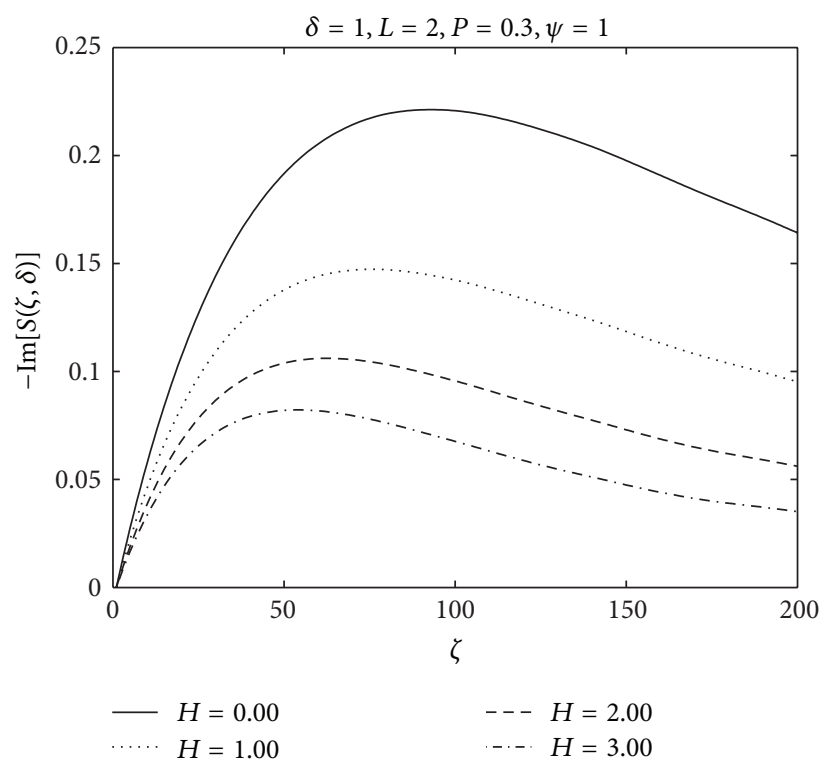

(b)

Figure 7: Velocity profiles for different values of $H$.

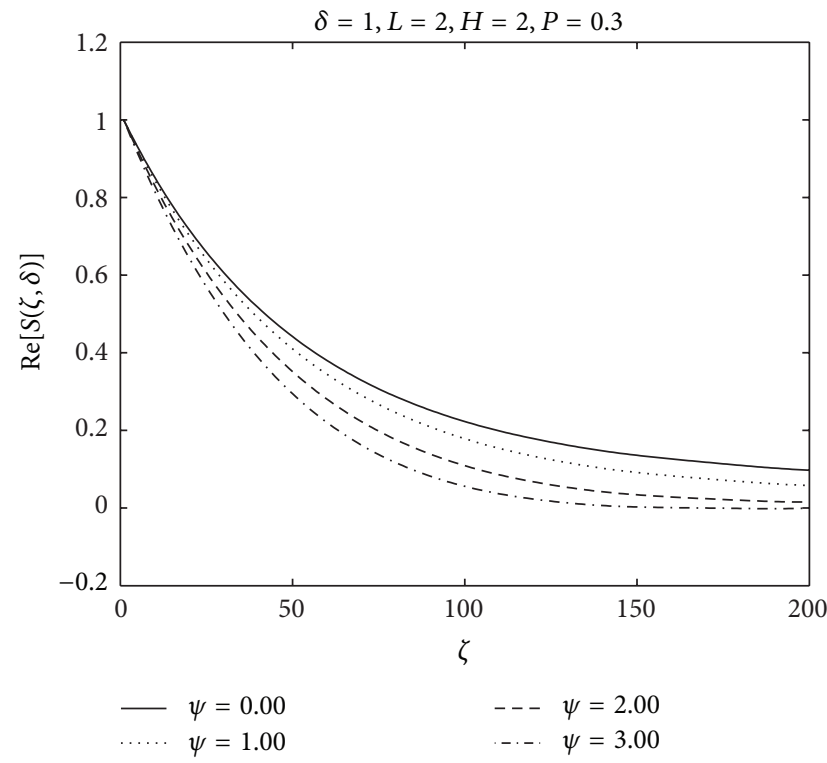

(a)

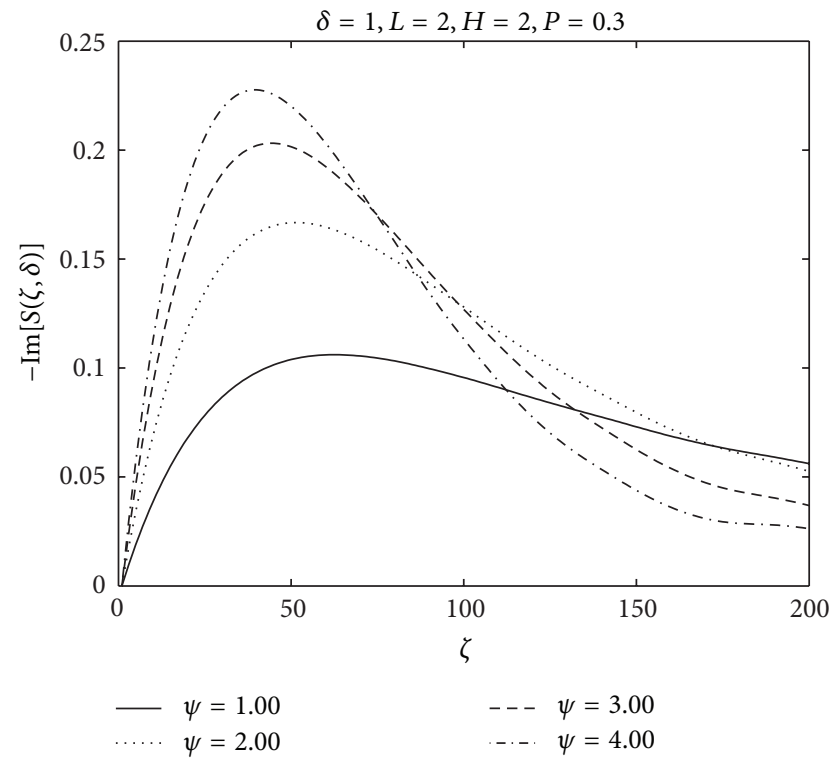

(b)

Figure 8: Velocity profiles for different values of $\psi$.

sketched in Figure 5. It is found that the magnitude of velocity components increases with dimensionless time. Further, it is observed that the variations of parameters in constant accelerated flow (i.e., Figures 1-5) are qualitatively similar (i.e., in Figure 6, the magnitudes of an imaginary component of velocity are decreasing function of $\beta$ ) to the effects plotted for variable accelerated flow (i.e., Figures 6-10). However, the velocity profiles in constant accelerated flow and variable accelerated flow are not similar quantitatively. Comparison shows that the velocity profiles in variable accelerated flow are larger when compared to those of constant accelerated flow.

\section{Conclusions}

In this research, the constant accelerated flow and variable accelerated flow of non-Newtonian fluid in a rotating frame are examined. The exact solutions are first established via 


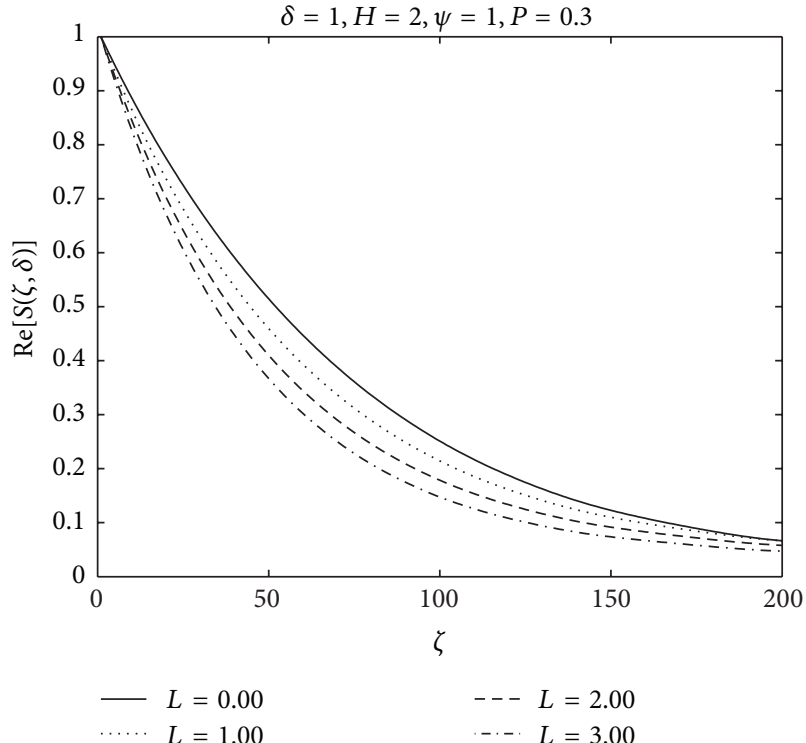

(a)

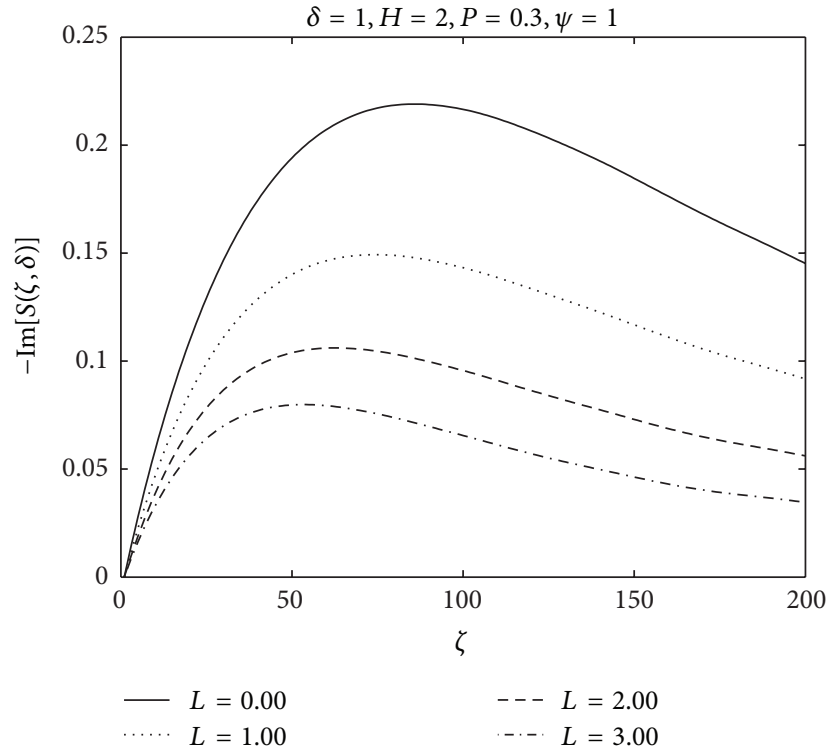

(b)

FIGURE 9: Velocity profiles for different values of $L$.

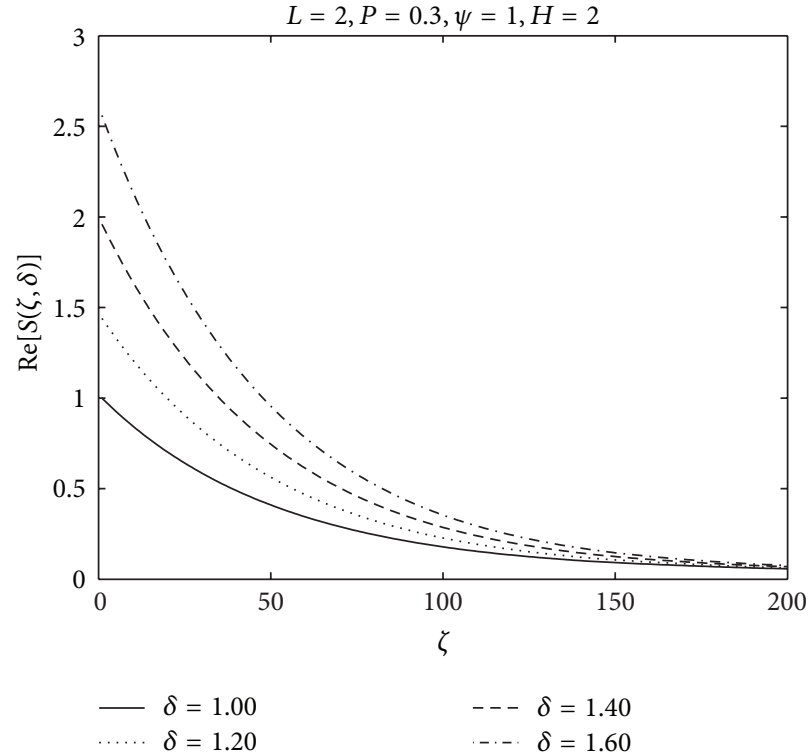

(a)

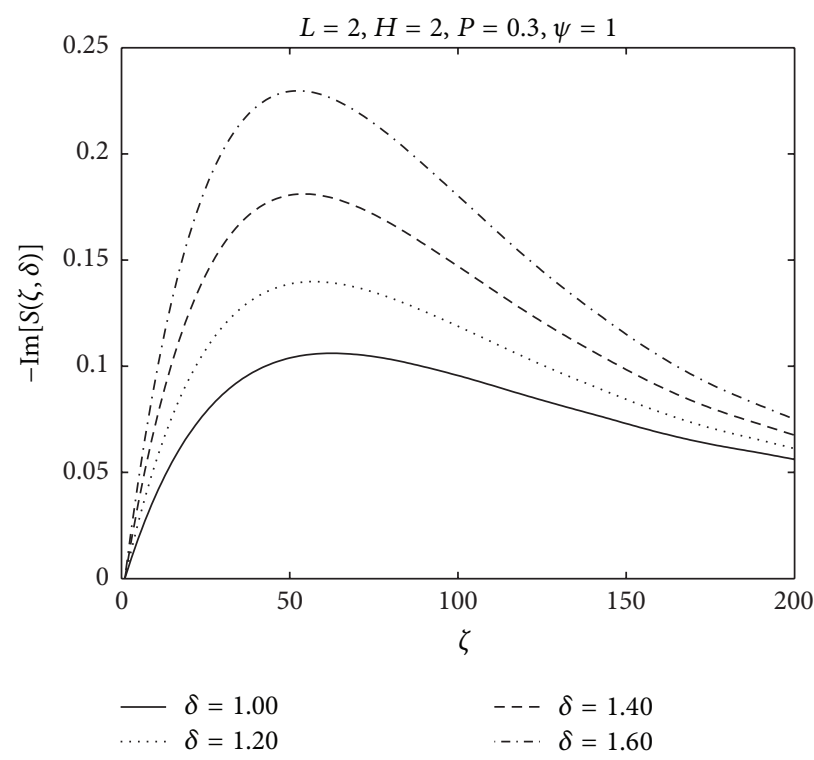

(b)

FIGURE 10: Velocity profiles for different values of $\delta$.

Fourier sine transform, and then the following observations have been noted through the graphs.

(i) The magnitude of velocity components in viscous fluid is greater when compared with Maxwell fluid in case of constant accelerated flow and variable accelerated flow. (ii) The magnitude of velocity components for constant accelerated flow and variable accelerated flow and MHD fluid is less than that of hydrodynamic fluid.

(iii) The behaviours of $B$ on the magnitude of velocity components are quite opposite to those of $M$ and $\beta$.

(iv) The salient features of the velocity profile with respect to variations of various embedded parameters in 
constant and variable accelerated cases are similar in a qualitative sense.

\section{Acknowledgments}

This research is partially funded by UTM RUG Vot. no. PY/2011/02418. Dr. Faisal is thankful to UTM for the postdoctoral fellowship.

\section{References}

[1] P. Puri, "Rotary flow of an elastico-viscous fluid on an oscillating plate," Journal of Applied Mathematics and Mechanics, vol. 54, no. 11, pp. 743-745, 1974.

[2] M. Hussain, T. Hayat, S. Asghar, and C. Fetecau, "Oscillatory flows of second grade fluid in a porous space," Nonlinear Analysis: Real World Applications, vol. 11, no. 4, pp. 2403-2414, 2010.

[3] C. Fetecau, S. C. Prasad, and K. R. Rajagopal, "A note on the flow induced by a constantly accelerating plate in an Oldroyd-B fluid," Applied Mathematical Modelling, vol. 31, no. 4, pp. 647654, 2007.

[4] W. Tan and T. Masuoka, "Stokes' first problem for a second grade fluid in a porous half-space with heated boundary," International Journal of Non-Linear Mechanics, vol. 40, no. 4, pp. 515-522, 2005.

[5] C. Fetecau, M. Athar, and C. Fetecau, "Unsteady flow of a generalized Maxwell fluid with fractional derivative due to a constantly accelerating plate," Computers and Mathematics with Applications, vol. 57, no. 4, pp. 596-603, 2009.

[6] M. Husain, T. Hayat, C. Fetecau, and S. Asghar, "On accelerated flows of an Oldroyd-B fluid in a porous medium," Nonlinear Analysis: Real World Applications, vol. 9, no. 4, pp. 1394-1408, 2008.

[7] M. Khan, E. Naheed, C. Fetecau, and T. Hayat, "Exact solutions of starting flows for second grade fluid in a porous medium," International Journal of Non-Linear Mechanics, vol. 43, no. 9, pp. 868-879, 2008.

[8] F. Salah, Z. A. Aziz, and D. L. C. Ching, "New exact solution for Rayleigh-Stokes problem of Maxwell fluid in a porous medium and rotating frame," Results in Physics, vol. 1, no. 1, pp. 9-12, 2011.

[9] M. Khan, M. Saleem, C. Fetecau, and T. Hayat, "Transient oscillatory and constantly accelerated non-Newtonian flow in a porous medium," International Journal of Non-Linear Mechanics, vol. 42, no. 10, pp. 1224-1239, 2007.

[10] F. Salah, Z. Abdul Aziz, and D. L. C. Ching, "New exact solutions for MHD transient rotating flow of a second-grade fluid in a porous medium," Journal of Applied Mathematics, vol. 2011, Article ID 823034, 8 pages, 2011.

[11] C. Fetecau, T. Hayat, M. Khan, and C. Fetecau, "Erratum: Unsteady flow of an Oldroyd-B fluid induced by the impulsive motion of a plate between two side walls perpendicular to the plate," Acta Mechanica, vol. 216, no. 1-4, pp. 359-361, 2011.

[12] C. Fetecau, T. Hayat, J. Zierep, and M. Sajid, "Energetic balance for the Rayleigh-Stokes problem of an Oldroyd-B fluid," Nonlinear Analysis: Real World Applications, vol. 12, no. 1, pp. 1-13, 2011.

[13] K. R. Rajagopal and A. S. Gupta, "On a class of exact solutions to the equations of motion of a second grade fluid," International Journal of Engineering Science, vol. 19, no. 7, pp. 1009-1014, 1981.
[14] M. E. Erdoğan and C. E. Imrak, "On unsteady unidirectional flows of a second grade fluid," International Journal of NonLinear Mechanics, vol. 40, no. 10, pp. 1238-1251, 2005.

[15] F. Salah, Z. A. Aziz, and D. L. C. Ching, "Accelerated flows of a magnetohydrodynamic (MHD) second grade fluid over an oscillating plate in a porous medium and rotating frame," International Journal of Physical Sciences, vol. 6, no. 36, pp. 8027-8035, 2011.

[16] C. Fetecau and C. Fetecau, "Starting solutions for some unsteady unidirectional flows of a second grade fluid," International Journal of Engineering Science, vol. 43, no. 10, pp. 781-789, 2005.

[17] T. Hayat, K. Hutter, S. Asghar, and A. M. Siddiqui, "MHD flows of an Oldroyd-B fluid," Mathematical and Computer Modelling, vol. 36, no. 9-10, pp. 987-995, 2002.

[18] T. Hayat, S. Nadeem, S. Asghar, and A. M. Siddiqui, "Fluctuating flow of a third-grade fluid on a porous plate in a rotating medium," International Journal of Non-Linear Mechanics, vol. 36, no. 6, pp. 901-916, 2001.

[19] S. Abelman, E. Momoniat, and T. Hayat, "Steady MHD flow of a third grade fluid in a rotating frame and porous space," Nonlinear Analysis: Real World Applications, vol. 10, no. 6, pp. 3322-3328, 2009. 


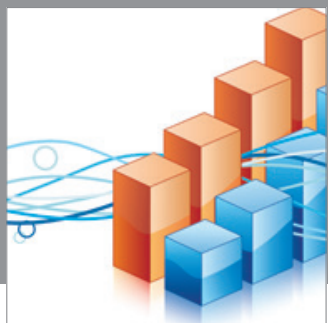

Advances in

Operations Research

mansans

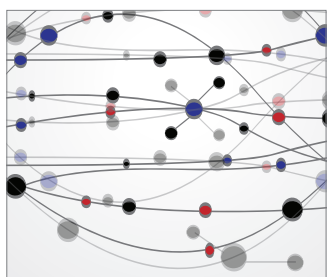

The Scientific World Journal
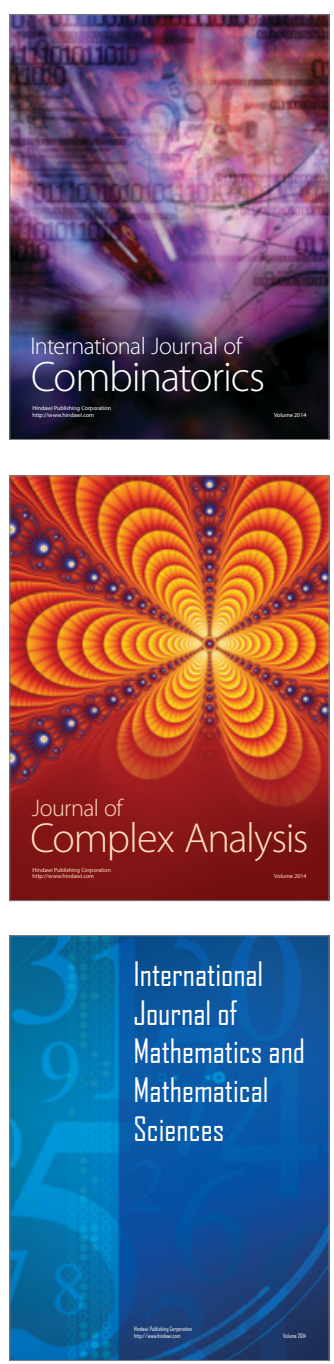
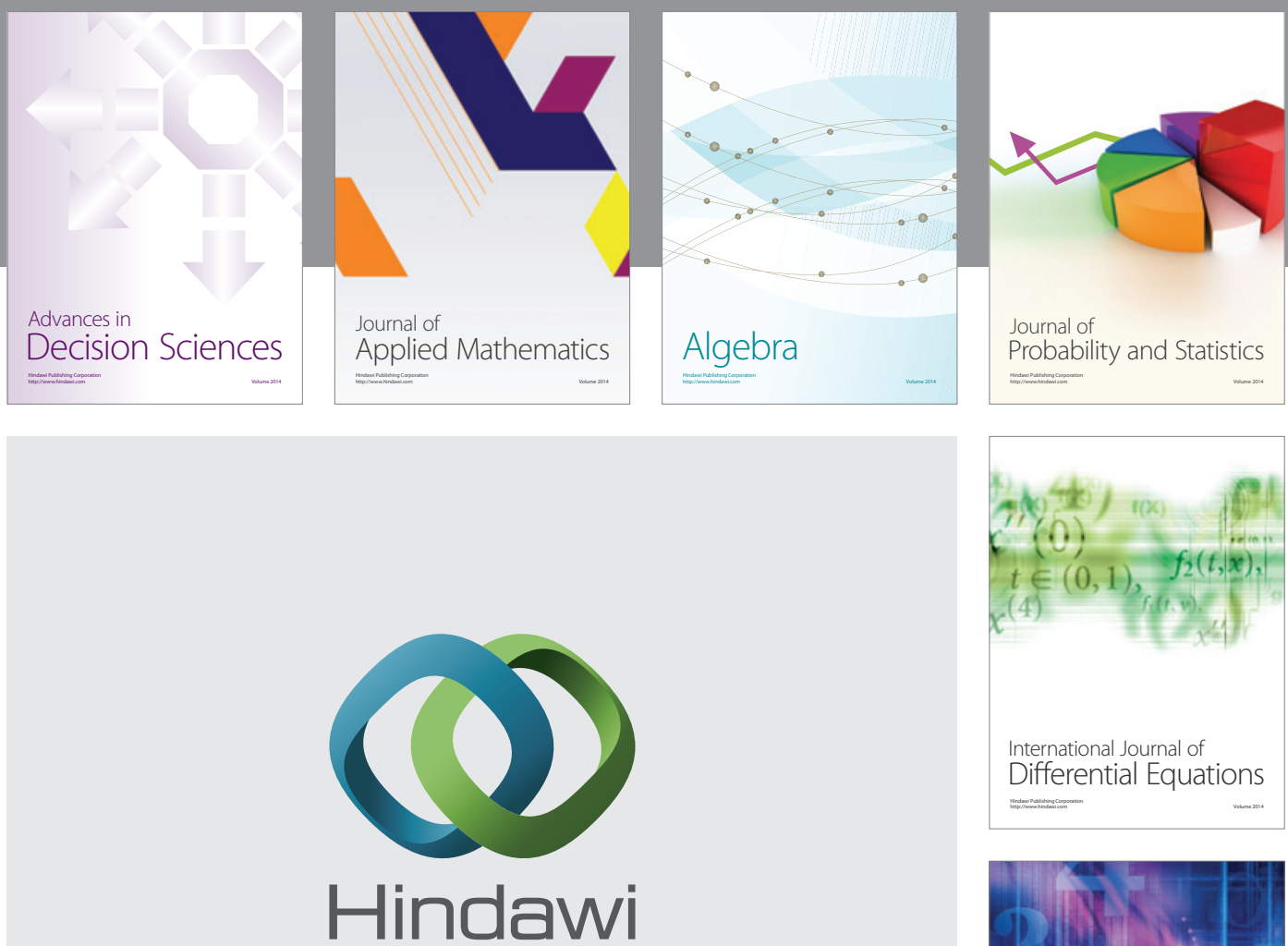

Submit your manuscripts at http://www.hindawi.com
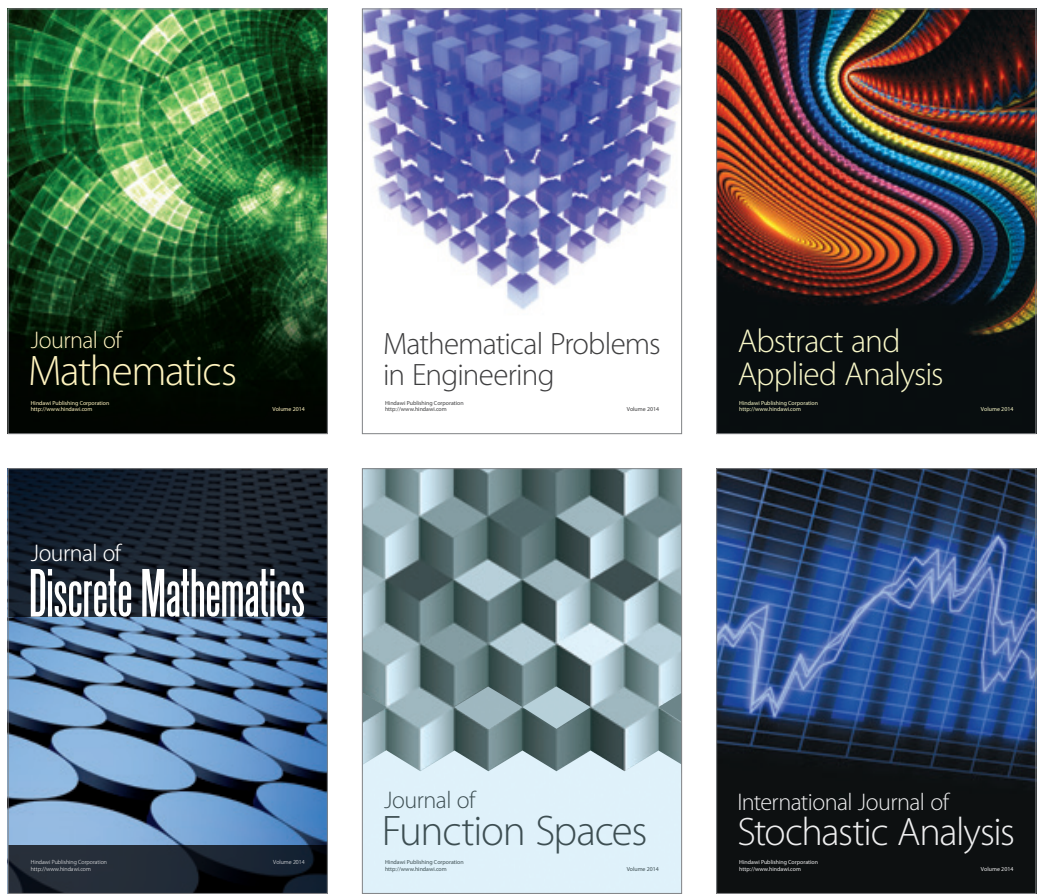

Journal of

Function Spaces

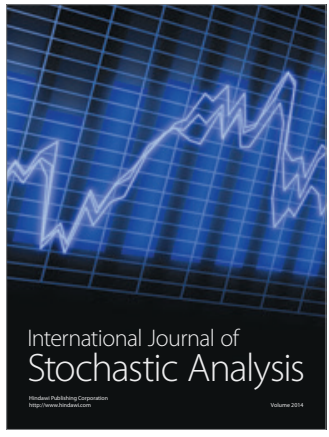

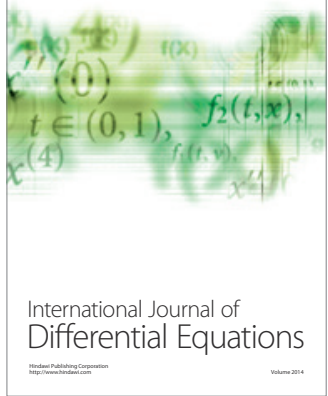
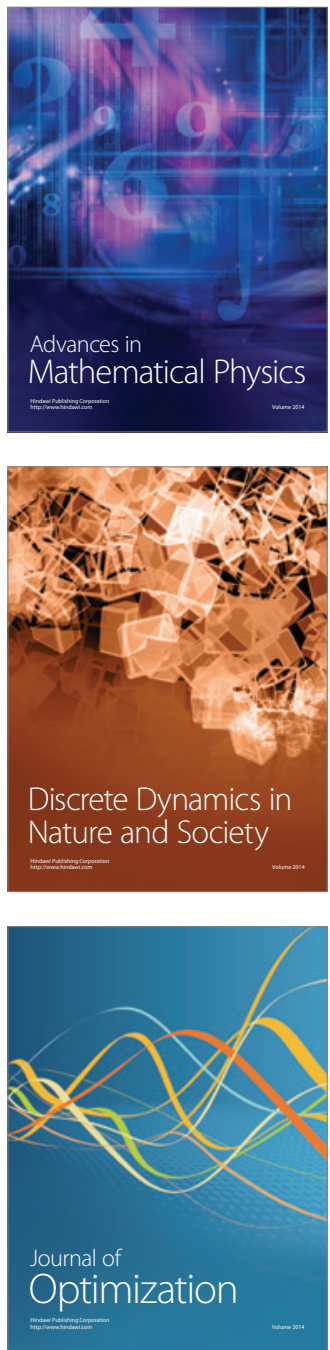